\title{
Asymptotic Approximation of Convex Curves
}

\author{
Monika Ludwig
}

\begin{abstract}
L. Fejes Tóth gave asymptotic formulae as $n \rightarrow \infty$ for the distance between a smooth convex disc and its best approximating inscribed or circumscribed polygons with at most $n$ vertices, where the distance is in the sense of the symmetric difference metric. In this paper these formulae are extended by specifying the second terms of the asymptotic expansions. Tools are from affine differential geometry.
\end{abstract}

\section{Introduction}

Let $C$ be a closed convex curve in the Euclidean plane $\mathbb{E}^{2}$ and let $\mathcal{P}_{n}^{i}(C)$ be the set of all convex polygons with at most $n$ vertices that are inscribed in $C$. We measure the distance of $C$ and $P_{n} \in \mathcal{P}_{n}^{i}(C)$ by the symmetric difference metric $\delta^{S}$ and study the asymptotic behaviour of

$$
\delta^{S}\left(C, \mathcal{P}_{n}^{i}\right)=\inf \left\{\delta^{S}\left(C, P_{n}\right): P_{n} \in \mathcal{P}_{n}^{i}(C)\right\}
$$

as $n \rightarrow \infty$. In case of circumscribed polygons the analogous notion is $\delta^{S}\left(C, \mathcal{P}_{n}^{c}\right)$.

For a $C \in \mathcal{C}^{2}$ with positive curvature function $\kappa(t)$, the following asymptotic formulae were given by L. Fejes Tóth [2], [3]

$$
\delta^{S}\left(C, \mathcal{P}_{n}^{i}\right) \sim \frac{1}{12}\left(\int_{0}^{l} \kappa^{1 / 3}(t) d t\right)^{3} \frac{1}{n^{2}} \quad \text { as } n \rightarrow \infty
$$

and

$$
\delta^{S}\left(C, \mathcal{P}_{n}^{c}\right) \sim \frac{1}{24}\left(\int_{0}^{l} \kappa^{1 / 3}(t) d t\right)^{3} \frac{1}{n^{2}} \quad \text { as } n \rightarrow \infty,
$$

where $t$ is the arc length and $l$ the length of $C$. Complete proofs of these results are due to D. E. McClure and R. A. Vitale [9].

In this article we extend these asymptotic formulae by deriving the second terms in the asymptotic expansions. For a $C \in \mathcal{C}^{4}$ these terms are of order $1 / n^{4}$ and depend on the affine curvature of the curve.

Besides the above mentioned results several other asymptotic formulae for the distance of a convex body to its best approximating polytopes are known. In the case of the symmetric difference metric and inscribed and circumscribed polytopes these formulae were derived by P. M. Gruber in [5], [6], and [8] for any dimension; see also [10]. For detailed information see the surveys [4] and [7]. 


\section{Some Tools from Affine Differential Geometry}

$\delta^{S}\left(C, \mathcal{P}_{n}^{i}\right)$ and $\delta^{S}\left(C, \mathcal{P}_{n}^{c}\right)$ are invariant with respect to area-preserving affine transformations. Therefore, we choose the affine arc length to be the parameter of $C$.

Let $C \in \mathcal{C}^{2}$ and let the curvature of $C$ be positive. Then the affine arc length is given by

$$
s(t)=\int_{0}^{t} \kappa^{1 / 3}(\tau) d \tau, 0 \leq t \leq l,
$$

where $t$ is the ordinary arc length and $\kappa(t)$ the curvature of $C$. The affine length $\lambda$ of $C$ is

$$
\lambda=\int_{0}^{l} \kappa^{1 / 3}(\tau) d \tau
$$

In the following, let $x(s)$ be an affine arclength parametrization of $C$.

The affine curvature of $C$ is then given by

$$
k(s)=\left|x^{\prime \prime}(s), x^{\prime \prime \prime}(s)\right|
$$

where ' denotes differentiation with respect to affine arc length. It determines a curve up to an area-preserving affine transformation. Note that

$$
\left|x^{\prime}(s), x^{\prime \prime}(s)\right|=1 \text { and }\left|x^{\prime}(s), x^{\prime \prime \prime}(s)\right|=0 .
$$

See [1].

\section{Asymptotic Expansion for $\delta^{S}\left(C, \mathcal{P}_{n}^{i}\right)$}

Using the notions and notation from affine differential geometry just introduced, we are able to formulate our main result.

Theorem 1 Let $C$ be a closed convex curve in $\mathbb{E}^{2}$ of class $\mathcal{C}^{4}$ with positive ordinary curvature. Then

$$
\delta^{S}\left(C, \mathcal{P}_{n}^{i}\right) \sim \frac{1}{12} \frac{\lambda^{3}}{n^{2}}-\frac{1}{2} \frac{\lambda^{4}}{5 !} \int_{0}^{\lambda} k(s) d s \frac{1}{n^{4}}+o\left(\frac{1}{n^{4}}\right)
$$

as $n \rightarrow \infty$.

For the proof we need the following two lemmas.

Lemma 1 For $0 \leq r \leq s \leq \lambda$, let $F(r, s)$ be the area of the piece between $C$ and the line segment with end points $x(r)$ and $x(s)$. Then

$$
F(r, s)=\frac{1}{2}\left(\frac{(s-r)^{3}}{3 !}-k(r) \frac{(s-r)^{5}}{5 !}+o\left((s-r)^{5}\right)\right)
$$

uniformly for all $0 \leq r \leq s \leq \lambda$ as $(s-r) \rightarrow 0$. 
Proof. Without loss of generality, let $r=0$. Since $C \in \mathcal{C}^{4}$, the affine arclength parametrization $x(s)$ of $C$ is of class $\mathcal{C}^{3}$. Let $\omega$ be a modulus of continuity function for $x^{\prime \prime \prime}(s)$; that is, $\omega$ is a continuous non-decreasing function on $[0, \infty]$ with $\omega(0)=0$ and such that

$$
\left\|x^{\prime \prime \prime}(s)-x^{\prime \prime \prime}(r)\right\| \leq \omega(|s-r|) \text { for all } r, s \in[0, \lambda] .
$$

Taylor's formula yields

$$
x^{\prime \prime}(s)=x^{\prime \prime}(0)+x^{\prime \prime \prime}(0) s+u_{1}(s),
$$

where $\left\|u_{1}(s)\right\| \leq s \omega(s)$. Integrating (6) twice we see that

$$
x^{\prime}(s)=x^{\prime}(0)+x^{\prime \prime}(0) s+x^{\prime \prime \prime}(0) \frac{s^{2}}{2}+u_{2}(s)
$$

and

$$
x(s)-x(0)=x^{\prime}(0) s+x^{\prime \prime}(0) \frac{s^{2}}{2}+x^{\prime \prime \prime}(0) \frac{s^{3}}{3 !}+u_{3}(s),
$$

where $\left\|u_{2}(s)\right\| \leq \frac{s^{2}}{2} \omega(s)$ and $\left\|u_{3}(s)\right\| \leq \frac{s^{3}}{3 !} \omega(s)$.

Since $x(s)$ is the affine arclength parametrization, (4), (5), (6), and (7) imply that

$$
1=\left|x^{\prime}(s), x^{\prime \prime}(s)\right|=1+\left|x^{\prime}(0), u_{1}(s)\right|+k(0) \frac{s^{2}}{2}+\alpha(s),
$$

where

$$
|\alpha(s)| \leq \mu_{1} \frac{s^{2}}{2} \omega(s)
$$

and $\mu_{1}$ is a constant which depends only on $C$. Rewriting (9) in the form

$$
\left|x^{\prime}(0), u_{1}(s)\right|=-k(0) \frac{s^{2}}{2}-\alpha(s)
$$

and integrating twice, we find that

$$
\left|x^{\prime}(0), u_{2}(s)\right|=-k(0) \frac{s^{3}}{3 !}-\beta(s),
$$

and

$$
\left|x^{\prime}(0), u_{3}(s)\right|=-k(0) \frac{s^{4}}{4 !}-\gamma(s),
$$

where $|\beta(s)| \leq \mu_{1} \frac{s^{3}}{3 !} \omega(s)$ and $|\gamma(s)| \leq \mu_{1} \frac{s^{4}}{4 !} \omega(s)$. Then combining (7), (8), (10), and (11), we obtain

$$
\left|x(s)-x(0), x^{\prime}(s)\right|=\frac{s^{2}}{2}-k(0) \frac{s^{4}}{4 !}+\delta(s),
$$


where

$$
|\delta(s)| \leq \mu_{2} s^{4} \omega(s)
$$

and $\mu_{2}$ is a constant which depends only on $C$.

Finally, integrating (12) gives

$$
F(0, s)=\frac{1}{2} \int_{0}^{s}\left|x(s)-x(0), x^{\prime}(s)\right| d s=\frac{1}{2}\left(\frac{s^{3}}{3 !}-k(0) \frac{s^{5}}{5 !}+\int_{0}^{s} \delta(s) d s\right) .
$$

Now, noting that $[0, \lambda]$ is compact and $\mu_{2}$ and $\omega$ depend only on $C$, the last equality together with (13) yields Lemma 1.

Lemma 2 Let $P_{n} \in \mathcal{P}_{n}^{i}(C), n=3,4, \ldots$, be a sequence of best approximating polygons of $C$. Let $x\left(s_{n i}\right), i=1, \ldots, n$, be the vertices of $P_{n}$. Define $\lambda_{n i}=$ $s_{n i}-s_{n, i-1}$. Then

$$
\lambda_{n i}=\frac{\lambda}{n}+o\left(\frac{1}{n}\right) \text { uniformly in } i \text { as } n \rightarrow \infty .
$$

Proof. Since $P_{n}$ is a best approximating, the line segment connecting $x\left(s_{n, i-1}\right)$ and $x\left(s_{n, i+1}\right)$ is parallel to the tangent at $x\left(s_{n i}\right)$, i. e.

$$
\left|x\left(s_{n, i+1}\right)-x\left(s_{n, i-1}\right), x^{\prime}\left(s_{n i}\right)\right|=0 \text { for } i=1, \ldots, n-1,
$$

see $[3]$.

We proceed by using Taylor's formula to derive expressions for the $\lambda_{n i}$ 's from (14). Let $\omega$ be a modulus of continuity function for $x^{\prime \prime \prime}(s)$. Then for given $n$ and $i$,

$$
x(s)=x\left(s_{n i}\right)+x^{\prime}\left(s_{n i}\right)\left(s-s_{n i}\right)+x^{\prime \prime}\left(s_{n i}\right) \frac{\left(s-s_{n i}\right)^{2}}{2}+x^{\prime \prime \prime}\left(s_{n i}\right) \frac{\left(s-s_{n i}\right)^{3}}{3 !}+u_{n i}(s),
$$

where

$$
\left\|u_{n i}(s)\right\| \leq \frac{\left|s-s_{n i}\right|^{3}}{3 !} \omega\left(s-s_{n i}\right) .
$$

Substituting $s_{n, i-1}$ and $s_{n, i+1}$ for $s$ and subtracting, we find that

$$
\begin{aligned}
x\left(s_{n, i+1}\right)-x\left(s_{n, i-1}\right)= & x^{\prime}\left(s_{n i}\right)\left(\lambda_{n i}+\lambda_{n, i+1}\right)+\frac{x^{\prime \prime}\left(s_{n i}\right)}{2}\left(\lambda_{n, i+1}^{2}-\lambda_{n i}^{2}\right)+ \\
& +\frac{x^{\prime \prime \prime}\left(s_{n i}\right)}{3 !}\left(\lambda_{n i}^{3}+\lambda_{n, i+1}^{3}\right)-u_{n i}\left(s_{n, i-1}\right)+u_{n i}\left(s_{n, i+1}\right) .
\end{aligned}
$$

Combining this with (14), we obtain

$$
\left|x\left(s_{n, i+1}\right)-x\left(s_{n, i-1}\right), x^{\prime}\left(s_{n i}\right)\right|=
$$




$$
=\frac{\lambda_{n i}^{2}}{2}-\frac{\lambda_{n, i+1}^{2}}{2}+\left|x^{\prime}\left(s_{n i}\right), u_{n i}\left(s_{n, i-1}\right)\right|-\left|x^{\prime}\left(s_{n i}\right), u_{n i}\left(s_{n, i+1}\right)\right|=0 .
$$

This can be rewritten as

$$
\lambda_{n i}^{2}=\lambda_{n, i+1}^{2}+2\left|x^{\prime}\left(s_{n i}\right), u_{n i}\left(s_{n, i+1}\right)-u_{n i}\left(s_{n, i-1}\right)\right|
$$

for $i=1, \ldots, n-1$. Thus

$$
\lambda_{n i}^{2}=\lambda_{n j}^{2}+2 \sum_{k=i}^{j-1}\left|x^{\prime}\left(s_{n k}\right), u_{n k}\left(s_{n, k+1}\right)-u_{n k}\left(s_{n, k-1}\right)\right|
$$

for $i<j \leq n$ and

$$
\lambda_{n i}^{2}=\lambda_{n j}^{2}-2 \sum_{k=j}^{i-1}\left|x^{\prime}\left(s_{n k}\right), u_{n k}\left(s_{n, k+1}\right)-u_{n k}\left(s_{n, k-1}\right)\right|
$$

for $1 \leq j<i$. Summing on $j$ from $i+1$ to $n$ in (16) and from 1 to $i-1$ in (17), we obtain

$$
\begin{aligned}
(n-i) \lambda_{n i}^{2} & =\sum_{j=i+1}^{n} \lambda_{n j}^{2}+2 \sum_{j=i+1}^{n} \sum_{k=i}^{j-1}\left|x^{\prime}\left(s_{n k}\right), u_{n k}\left(s_{n, k+1}\right)-u_{n k}\left(s_{n, k-1}\right)\right| \\
(i-1) \lambda_{n i}^{2} & =\sum_{j=1}^{i-1} \lambda_{n j}^{2}-2 \sum_{j=1}^{i-1} \sum_{k=j}^{i-1}\left|x^{\prime}\left(s_{n k}\right), u_{n k}\left(s_{n, k+1}\right)-u_{n k}\left(s_{n, k-1}\right)\right| .
\end{aligned}
$$

Adding $\lambda_{n i}^{2}$ to the sum of these equations gives

$$
n \lambda_{n i}^{2}=\sum_{j=1}^{n} \lambda_{n j}^{2}+\alpha_{n i},
$$

where

$$
\begin{aligned}
\alpha_{n i}= & 2 \sum_{j=i+1}^{n} \sum_{k=i}^{j-1}\left|x^{\prime}\left(s_{n k}\right), u_{n k}\left(s_{n, k+1}\right)-u_{n k}\left(s_{n, k-1}\right)\right|- \\
& -2 \sum_{j=1}^{i-1} \sum_{k=j}^{i-1}\left|x^{\prime}\left(s_{n k}\right), u_{n k}\left(s_{n, k+1}\right)-u_{n k}\left(s_{n, k-1}\right)\right| .
\end{aligned}
$$

Multiplying (18) by $n$ gives the desired expressions for the $\lambda_{n i}$ 's:

$$
n^{2} \lambda_{n i}^{2}=n \sum_{j=1}^{n} \lambda_{n j}^{2}+n \alpha_{n i}
$$

We will study the behaviour of these equations as $n \rightarrow \infty$. For the right-hand side of (19) we will show that

$$
\lim _{n \rightarrow \infty} n \sum_{i=1}^{n} \lambda_{n i}^{2}=\lambda^{2}
$$


and

$$
\lim _{n \rightarrow \infty} n \max _{i=1, \ldots, n}\left|\alpha_{n i}\right|=0 .
$$

In order to prove (20) we rewrite the inequalities

$$
\frac{\lambda}{n}=\frac{1}{n} \sum_{i=1}^{n} \lambda_{n i} \leq\left(\frac{1}{n} \sum_{i=1}^{n} \lambda_{n i}^{2}\right)^{1 / 2} \leq\left(\frac{1}{n} \sum_{i=1}^{n} \lambda_{n i}^{3}\right)^{1 / 3},
$$

in the form

$$
\lambda^{2} \leq n \sum_{i=1}^{n} \lambda_{n i}^{2} \leq\left(n^{2} \sum_{i=1}^{n} \lambda_{n i}^{3}\right)^{2 / 3} .
$$

As a consequence of Lemma 1,

$$
F_{n i}=\frac{\lambda_{n i}^{3}}{12}+o\left(\lambda_{n i}^{3}\right) \quad \text { uniformly as } \lambda_{n i} \rightarrow 0 .
$$

Hence, recalling the asymptotic formula (1)

$$
\lim _{n \rightarrow \infty} n^{2} \delta^{S}\left(C, \mathcal{P}_{n}^{i}\right)=\lim _{n \rightarrow \infty} n^{2} \sum_{i=1}^{n} F_{n i}=\frac{\lambda^{3}}{12},
$$

we obtain

$$
\lim _{n \rightarrow \infty} n^{2} \sum_{i=1}^{n} \lambda_{n i}^{3}=\lambda^{3}
$$

and (20) follows from (22).

Next we establish (21). Define $\nu=\sup _{0 \leq s \leq \lambda}\left\|x^{\prime}(s)\right\|$. Then (15) gives

$$
\left\|x^{\prime}\left(s_{n i}\right), u_{n i}\left(s_{n, i-1}\right)\right\| \leq \frac{\nu}{3 !} \lambda_{n i}^{3} \omega\left(\lambda_{n i}\right) .
$$

The definition of the $\alpha_{n i}$ 's implies that

$$
\left|\alpha_{n i}\right| \leq 2 n \sum_{k=1}^{n}\left\|x^{\prime}\left(s_{n k}\right), u_{n k}\left(s_{n, k+1}\right)-u_{n k}\left(s_{n, k-1}\right)\right\| .
$$

Combining this with (24), we have

$$
n\left|\alpha_{n i}\right| \leq 4 n^{2} \sum_{k=1}^{n} \frac{\nu}{3 !} \lambda_{n i}^{3} \omega\left(\lambda_{n i}\right) \leq 4 \frac{\nu}{3 !} \omega\left(\max _{i=1, \ldots, n} \lambda_{n i}\right) n^{2} \sum_{k=1}^{n} \lambda_{n i}^{3} .
$$

Since $\delta^{S}\left(C, \mathcal{P}_{n}^{i}\right) \rightarrow 0, \lim _{n \rightarrow \infty} \max _{i=1, \ldots, n} \lambda_{n i}=0$. Hence (21) follows from (23) and $(25)$.

By (20) and (21), it follows from (19) that

$$
\lim _{n \rightarrow \infty} n^{2} \max _{i=1, \ldots, n} \lambda_{n i}^{2}=\lim _{n \rightarrow \infty} n^{2} \min _{i=1, \ldots, n} \lambda_{n i}^{2}=\lambda^{2},
$$


which proves the lemma.

Now we are able to prove the main theorem.

Proof of Theorem 1. First we choose for $n=3,4, \ldots$ polygons $Q_{n}$ with vertices at the points $x\left(i \frac{\lambda}{n}\right), i=0,1, \ldots, n$, and denote the area of the $i$-th piece between $C$ and $P_{n}$ by $F_{n i}$. Let $\varepsilon>0$ be chosen. Lemma 1 shows that there is an integer $n_{0}$ such that

$$
F_{n i} \leq \frac{1}{2}\left(\frac{1}{3 !} \frac{\lambda^{3}}{n^{3}}-\frac{1}{5 !} k\left(i \frac{\lambda}{n}\right) \frac{\lambda^{5}}{n^{5}}+\varepsilon \frac{\lambda^{5}}{n^{5}}\right)
$$

for all $n \geq n_{0}$. Hence

$$
\begin{aligned}
\delta^{S}\left(C, \mathcal{P}_{n}^{i}\right)-\frac{1}{12} \frac{\lambda^{3}}{n^{2}} & \leq \delta^{S}\left(C, P_{n}\right)-\frac{1}{12} \frac{\lambda^{3}}{n^{2}} \\
& \leq \sum_{i=1}^{n} F_{n i}-\frac{1}{12} \frac{\lambda^{3}}{n^{2}} \\
& \leq \frac{1}{2}\left(\sum_{i=1}^{n}\left(\frac{1}{3 !} \frac{\lambda^{3}}{n^{3}}-\frac{1}{5 !} k\left(i \frac{\lambda}{n}\right) \frac{\lambda^{5}}{n^{5}}+\varepsilon \frac{\lambda^{5}}{n^{5}}\right)-\frac{1}{3 !} \frac{\lambda^{3}}{n^{2}}\right)= \\
& =\frac{1}{2}\left(\sum_{i=1}^{n}\left(-\frac{1}{5 !} k\left(i \frac{\lambda}{n}\right) \frac{\lambda^{5}}{n^{5}}\right)+\frac{\lambda^{5}}{n^{5}} \varepsilon\right)= \\
& =-\frac{1}{2} \frac{\lambda^{4}}{5 !} \frac{1}{n^{4}} \sum_{i=1}^{n} k\left(i \frac{\lambda}{n}\right) \frac{\lambda}{n}+\frac{1}{2} \frac{\lambda^{5}}{n^{4}} \varepsilon
\end{aligned}
$$

Since $\varepsilon>0$ was arbitrary,

$$
\limsup _{n \rightarrow \infty} n^{4}\left(\delta^{S}\left(C, \mathcal{P}_{n}^{i}\right)-\frac{1}{12} \frac{\lambda^{3}}{n^{2}}\right) \leq-\frac{1}{2} \frac{\lambda^{4}}{5 !} \int_{0}^{\lambda} k(s) d s .
$$

In order to show the opposite inequality, let $P_{n}$ be a sequence of best approximating polygons. Let $x\left(s_{n i}\right)$ be the vertices of $P_{n}$ and let $\lambda_{n i}=s_{n i}-s_{n, i-1}$. Choose $\varepsilon>0$. Then by Lemma 1 , there is an integer $n_{0}$ such that

$$
F_{n i} \geq \frac{1}{2}\left(\frac{\lambda_{n i}^{3}}{3 !}-k\left(s_{n, i-1}\right) \frac{\lambda_{n i}^{5}}{5 !}-\varepsilon \lambda_{n i}^{5}\right)
$$

for all $n \geq n_{0}$. Using the inequality

$$
\frac{1}{n^{2}} \lambda^{3}=\frac{1}{n^{2}}\left(\sum_{i=1}^{n} \lambda_{n i}\right)^{3} \leq \sum_{i=1}^{n} \lambda_{n i}^{3},
$$

we see that

$$
\begin{aligned}
n^{4}\left(\delta^{S}\left(C, \mathcal{P}_{n}^{i}\right)-\frac{1}{12} \frac{\lambda^{3}}{n^{2}}\right) & \geq n^{4}\left(\sum_{i=1}^{n} F_{n i}-\frac{1}{12} \sum_{i=1}^{n} \lambda_{n i}^{3}\right) \\
& \geq \frac{n^{4}}{2} \sum_{i=1}^{n}\left(\frac{\lambda_{n i}^{3}}{3 !}-k\left(s_{n, i-1}\right) \frac{\lambda_{n i}^{5}}{5 !}-\varepsilon \lambda_{n i}^{5}-\frac{\lambda_{n i}^{3}}{3 !}\right)= \\
& =\frac{n^{4}}{2} \sum_{i=1}^{n}\left(-k\left(s_{n, i-1}\right) \frac{\lambda_{n i}^{5}}{5 !}-\varepsilon \lambda_{n i}^{5}\right) .
\end{aligned}
$$


By Lemma 2

$$
\lambda_{n i}=\frac{\lambda}{n}+o\left(\frac{1}{n}\right) \quad \text { uniformly in } i .
$$

Thus

$$
\begin{aligned}
\frac{n^{4}}{2} \sum_{i=1}^{n}\left(-k\left(s_{n, i-1}\right) \frac{\lambda_{n i}^{5}}{5 !}-\varepsilon \lambda_{n i}^{5}\right) & =\frac{n^{4}}{2} \sum_{i=1}^{n}\left(-\frac{k\left(s_{n, i-1}\right)}{5 !} \frac{\lambda^{5}}{n^{5}}+o\left(\frac{1}{n^{5}}\right)-\varepsilon \frac{\lambda^{5}}{n^{5}}\right) \\
& =-\frac{1}{2} \frac{\lambda^{4}}{5 !} \sum_{i=1}^{n}\left(k\left(s_{n, i-1}\right) \frac{\lambda}{n}\right)-\varepsilon \frac{\lambda^{5}}{2}+o(1)= \\
& =-\frac{1}{2} \frac{\lambda^{4}}{5 !} \sum_{i=1}^{n} k\left(s_{n, i-1}\right) \lambda_{n i}-\varepsilon \frac{\lambda^{5}}{2}+o(1) .
\end{aligned}
$$

Since $\varepsilon>0$ was arbitrary, we have

$$
\liminf _{n \rightarrow \infty} n^{4}\left(\delta^{S}\left(C, \mathcal{P}_{n}^{i}\right)-\frac{1}{12} \frac{\lambda^{3}}{n^{2}}\right) \geq-\frac{1}{2} \frac{\lambda^{4}}{5 !} \int_{0}^{\lambda} k(s) d s
$$

This together with (26) concludes the proof of Theorem 1.

\section{Asymptotic Expansion for $\delta\left(C, \mathcal{P}_{n}^{c}\right)$}

Theorem 2 Let $C$ be a closed convex curve in $\mathbb{E}^{2}$ of class $\mathcal{C}^{4}$ with positive ordinary curvature. Then

$$
\delta^{S}\left(C, \mathcal{P}_{n}^{c}\right) \sim \frac{1}{24} \frac{\lambda^{3}}{n^{2}}+\frac{1}{2} \frac{\lambda^{4}}{5 !} \int_{0}^{\lambda} k(s) d s \frac{1}{n^{4}}+o\left(\frac{1}{n^{4}}\right)
$$

as $n \rightarrow \infty$.

The proof is analogous to that of Theorem 1. As a tool we use the property, that the edges of best approximating circumscribed polygons of $C$ touch $C$ at their midpoints (cf. [3]). Thus instead of (14) we have the equality

$$
\frac{\left|x\left(s_{n, i-1}\right)-x\left(s_{n i}\right), x^{\prime}\left(s_{n, i-1}\right)\right|}{\left|x^{\prime}\left(s_{n, i-1}\right), x^{\prime}\left(s_{n i}\right)\right|}=\frac{\left|x\left(s_{n, i+1}\right)-x\left(s_{n i}\right), x^{\prime}\left(s_{n, i+1}\right)\right|}{\left|x^{\prime}\left(s_{n i}\right), x^{\prime}\left(s_{n, i+1}\right)\right|} .
$$

\section{Acknowledgements}

I am obliged to Professor Gruber for many valuable suggestions and to Professor Chalk for his helpful comments. 


\section{References}

[1] W. Blaschke, Vorlesungen über Differentialgeometrie II, Springer, Berlin 1923

[2] L. Fejes Tóth, Approximation by polygons and polyhedra, Bull. Amer. Math. Soc. 4 (1948), 431-438

[3] L. Fejes Tóth, Lagerungen in der Ebene, auf der Kugel und im Raum, Springer, Berlin 1953, 1972

[4] P. M. Gruber, Approximation of convex bodies, In: Convexity and its applications (ed. by P. M. Gruber and J. M. Wills), Birkhäuser, Basel 1983

[5] P. M. Gruber, Volume approximation of convex bodies by inscribed polytopes, Math. Ann. 281 (1988), 229-245

[6] P. M. Gruber, Volume approximation of convex bodies by circumscribed polytopes, In: Applied Geometry and discrete Mathematics. The Victor Klee Festschrift, DIMACS Ser., Vol. 4 (Amer. Math. Soc., Providence, RI) (1991), 309-317

[7] P. M. Gruber, Aspects of approximation of convex bodies, In: Handbook of convex geometry (ed. by P. M. Gruber and J. M. Wills), North-Holland, Amsterdam 1993

[8] P. M. Gruber, Asymptotic estimates for best and stepwise approximation of convex bodies II, Forum Math., to appear

[9] D. E. McClure and R. A. Vitale, Polygonal approximation of plane convex bodies, J. Math. Anal. Appl. 51 (1975), 326-358

[10] R. Schneider, Affine-invariant approximation by convex polytopes, Stud. Sci. Math. Hung. 21 (1986), 401-408

Monika Ludwig

Abteilung für Analysis

Technische Universität Wien

Wiedner Hauptstraße 8-10/1142

A-1040 Wien 